\title{
A longitudinal study of depression and gestational diabetes in pregnancy and the postpartum period
}

\author{
Stefanie N. Hinkle ${ }^{1}$ - Germaine M. Buck Louis ${ }^{2} \cdot$ Shristi Rawal $^{1} \cdot$ Yeyi Zhu $^{1}$ • \\ Paul S. Albert ${ }^{3} \cdot$ Cuilin Zhang ${ }^{1}$
}

Received: 25 May 2016 / Accepted: 3 August 2016/Published online: 19 September 2016

(C) Springer-Verlag (outside the USA) 2016

\begin{abstract}
Aims/hypothesis Depression and glucose intolerance commonly co-occur among non-pregnant individuals; however, the temporal relationship between gestational diabetes (GDM) and depression during pregnancy and the postpartum period is less understood. Our objective was to assess longitudinal associations between depression early in pregnancy and GDM risk, as well as GDM and subsequent risk of postpartum depression.

Methods Data came from the prospective National Institute of Child Health and Human Development Fetal Growth Studies-Singleton cohort (2009-2013), and had been collected at 12 US clinical centres. Pregnant women without psychiatric disorders, diabetes or other chronic conditions before pregnancy were followed throughout pregnancy $(n=2477)$. Only women with GDM and matched controls were followed up at 6 weeks postpartum $(n=162)$. GDM was ascertained by a review of the medical records. Depression was assessed in the first (8-13 gestational weeks) and second (16-22 weeks) trimesters
\end{abstract}

Cuilin Zhang

zhangcu@mail.nih.gov

1 Epidemiology Branch, Division of Intramural Population Health Research, Eunice Kennedy Shriver National Institute of Child Health and Human Development, National Institutes of Health, 6710B Rockledge Drive, MSC 7004, Bethesda, MD 20817, USA

2 Office of the Director, Division of Intramural Population Health Research, Eunice Kennedy Shriver National Institute of Child Health and Human Development, National Institutes of Health, Bethesda, MD, USA

3 Biostatistics and Bioinformatics Branch, Division of Intramural Population Health Research, Eunice Kennedy Shriver National Institute of Child Health and Human Development, National Institutes of Health, Bethesda, MD, USA and at 6 weeks postpartum using the Edinburgh Postnatal Depression Scale. Postpartum depression was defined as a depressive symptom score $\geq 10$ or antidepressant medicine use after delivery. RR and $95 \% \mathrm{CI}$ were adjusted for prepregnancy BMI and other risk factors. GDM was considered to be the outcome for the first set of analyses, with depression in the first and second trimesters as the exposures. Postpartum depression was considered as the outcome for the second set of analyses, with GDM as the exposure.

Results Overall, comparing the highest and lowest quartiles of first-trimester depression scores, the scores from the highest quartile were associated with a significant twofold $(95 \% \mathrm{CI}$ $1.06,3.78$ ) increased risk of GDM, but this was attenuated to 1.72 -fold $(95 \%$ CI $0.92,3.23)$ after adjustment; the secondtrimester results were similar. The risk was stronger and significant in both trimesters among non-obese women ( $p$ for trend 0.02 and 0.01 , respectively), but null for obese women. Women with persistently high depression scores in both trimesters had the greatest risk of GDM (highest vs lowest quartile in both trimesters: adjusted RR 3.21, 95\% CI 1.00, 10.28). GDM was associated with an adjusted 4.62 -fold $(95 \%$ CI $1.26,16.98$ ) increased risk of subsequent postpartum depression.

Conclusions/interpretation This prospective study demonstrates a modest association between depressive symptoms early in pregnancy and an increased risk of incident GDM, as well as between GDM and subsequent postpartum depression risk, highlighting pregnancy and the postpartum period as an important susceptible time window during the life course for the interplay between depression and glucose intolerance phenotypes. GDM risk associated with elevated depressive symptoms was particularly high among non-obese women and women with symptoms persisting across the first two trimesters of pregnancy. 
Keywords Depression · Gestational diabetes · Postpartum . Pregnancy

\author{
Abbreviations \\ GDM Gestational diabetes mellitus \\ NICHD National Institute of Child Health and Human \\ Development
}

\section{Introduction}

More than one in four US women of reproductive age suffer from some degree of depressive symptoms [1]. Depression and glucose intolerance phenotypes are highly comorbid among non-pregnant individuals, with a bidirectional relation reported between the two conditions [2-5]. Depression is positively associated with metabolic perturbations, such as increased oxidative stress, chronic inflammation and insulin resistance, which can subsequently contribute to the development of hyperglycaemia [6]. On the other hand, depressive symptoms may result from hyperglycaemia, potentially via two pathways: the first is directly related to increased oxidative stress, inflammation or leptin resistance induced by hyperglycaemia, and the second results from increased psychological and physical stress caused by the management and/ or treatment of the diabetes [7]. Pregnancy is a state of insulin resistance. During pregnancy and in the postpartum period, women have an increased vulnerability for both depression and impaired glucose tolerance. However, this window of time is often overlooked as a critical time for the co-occurrence of these two conditions [8], which affects not only the woman herself, but also the health and well-being of her developing offspring.

Studies examining the temporal relations between depression and gestational diabetes (GDM) using longitudinal measures of depression are limited and inconsistent. The directionality and strength of the associations also remains unclear. Two studies have assessed the association between a history of depression prior to pregnancy and GDM and found inconsistent results $[9,10]$. One study reported a positive association between first-trimester depression and GDM, but this was attenuated after adjustment for pre-pregnancy BMI and total gestational weight gain, the latter including weight gain both before and after the diagnosis of GDM, making interpretation of the adjusted results unclear [11]. There has also been conflicting evidence on whether GDM is a risk factor for postpartum depression. Of the studies that have prospectively examined this association, the findings have either been conflicting [12-16] or have not distinguished between GDM and preexisting type 2 diabetes [16].
To address these critical knowledge gaps, we assessed the temporal relations between depression and GDM during the antenatal and postpartum period based on a prospective, multiracial cohort of women without psychiatric disorders or diabetes before pregnancy and a longitudinal assessment of depression. This study comprised two analyses. First, we examined the association between symptoms of depression early in pregnancy and the risk of incident GDM. Second, we examined the chronological association between GDM and subsequent risk of postpartum depression.

\section{Methods}

Study population This study was conducted among participants of the Eunice Kennedy Shriver National Institute of Child Health and Human Development (NICHD) Fetal Growth Studies-Singleton Cohort with low-risk pregnancies, and included 2334 non-obese [17] and 468 obese women $(n=2802$ in total). Women without pre-existing chronic diseases or medical conditions, including psychiatric disorders or diabetes before pregnancy, were enrolled at 12 US clinical centres (2009-2013). Women were enrolled in gestational weeks $8-13$, with five study visits occurring throughout pregnancy. The first analysis of antenatal depression and risk of GDM was limited to women with available medical chart abstraction ( $n=2584 ; 92.2 \%)$ and no missing data on critical variables $(n=2477 ; 88.4 \%)$. The second analysis, on GDM and postpartum depression, was limited to a subset of women with GDM $(n=81)$ and controls matched 1:1 in terms of age, race/ethnicity and study site $(n=81)$, with postpartum followup at 6 weeks. Institutional review board approval was obtained for all participating clinical sites, the data coordinating centre and NICHD.

Exposures and outcomes Depressive symptoms in the previous 7 days were assessed at study visits targeted at $8-13$ weeks (first trimester), 16-22 weeks (second trimester) and 6 weeks postpartum using the validated, 10-question self-rated Edinburgh Postnatal Depression Scale [18]. This scale is used clinically to screen for depression in pregnant and postpartum women as it excludes symptoms that are common in pregnancy (i.e. disrupted sleep patterns) [19]. At each visit, women self-reported their medication use. None of the women reported the use of antidepressant medication in the first trimester, and two women reported its use in the second trimester. Sensitivity analyses excluded these women.

Postpartum depression was classified using the standard suspected depression cut-off score of $\geq 10$ [18] or selfreported use of antidepressant medication after delivery. Fifteen women were considered to have postpartum depression, 13 were classified based on their depression symptom 
score, and five were currently taking medication for depression. Among the women with postpartum depression, most did not have suspected depression in the first or second trimester before the diagnosis of GDM $(n=10 ; 67 \%)$. We considered women taking postpartum antidepressant medication to be clinically diagnosed with depression; sensitivity analyses excluded these women from the postpartum depression classification.

GDM was diagnosed by a medical record review of OGTT results. As recommended by the American College of Obstetrics and Gynecologists [20], we classified GDM using the Carpenter and Coustan criteria of at least two diagnostic plasma glucose measurements at or above the defined thresholds (fasting, $5.3 \mathrm{mmol} / \mathrm{l} ; 1 \mathrm{~h}, 10.0 \mathrm{mmol} / \mathrm{l} ; 2 \mathrm{~h}, 8.6 \mathrm{mmol} / \mathrm{l} ; 3 \mathrm{~h}$, $7.8 \mathrm{mmol} / \mathrm{l}$ ). If OGTT results were not available but hospital discharge diagnosis indicated treatment of GDM by medication, the woman was considered as having had GDM $(n=12)$. In total, $107(4.3 \%)$ women with GDM were identified in the full cohort. Five women were diagnosed with GDM early in the second trimester before the ascertainment of depressive symptoms and were thus excluded from models assessing the association between second-trimester depression and GDM.

Covariates Considered covariates included well-documented factors related to depression or GDM, including age, race/ethnicity, parity, GDM during a prior pregnancy, prepregnancy BMI (calculated from self-reported weight and measured height at enrolment) and markers of socioeconomic status such as marital status, education and employment. We also considered social support, stress and gestational weight gain as potential confounding variables [21, 22]. Stress levels during the previous month were measured at enrolment using the 10-item Perceived Stress Scale [23]. Current social support was measured at enrolment using the 7-item ENRICHD Social Support Instrument [24]. Weight was measured at each study visit according to a standardised protocol. Gestational weight gain at the first- and second-trimester visits was calculated as the difference in weight measured at the respective visit minus the self-reported pre-pregnancy weight. As part of the inclusion criteria for the main study, non-obese women who smoked were not eligible for the study. At enrolment, obese women reported their smoking habits in the 6 months prior to pregnancy.

Statistical analysis The first set of analyses examined the association between depression early in pregnancy and incident risk of GDM. The RR of GDM was estimated using a log-Poisson regression model with robust variance estimates [25]. Depression scores were divided into quartiles, with the lowest quartile as the reference. The first models analysed first- and second-trimester depression scores separately. Next, the longitudinal association of elevated depressive symptoms across the first two trimesters and GDM risk was assessed by analysing the first and second trimesters together. Depression scores were categorised into five groups according to the highest quartile across the two trimesters: first quartile in both trimesters (reference), second quartile in either trimester, third quartile in either trimester, fourth quartile in either trimester, or fourth quartile in both trimesters. All analyses were estimated unadjusted and adjusted for age (continuous), race/ethnicity (non-Hispanic white, non-Hispanic black, Hispanic, Asian), pre-pregnancy BMI (continuous), education (high school or less, some college or associate degree, bachelor's degree or higher) and marital status (married or living with partner, single). Parity, employment, GDM in a prior pregnancy, social support and stress were not associated with GDM in this low-risk cohort.

Gestational weight gain in the first trimester may be a consequence of depression in the first trimester and was therefore not considered as a covariate for the regression models evaluating association of depression in the first trimester with risk of GDM. Furthermore, weight gain in the first trimester was not associated with subsequent depression in the second trimester and was thus not considered in the models of secondtrimester depression and GDM. Among the obese women, smoking was rare (3\%) and not associated with GDM, and was therefore not considered in models. We assessed for effect modification by pre-pregnancy obesity status (BMI $<30.0$ vs $\geq 30.0 \mathrm{~kg} / \mathrm{m}^{2}$ ) and race/ethnicity. The interaction between race/ethnicity and depression was not significant for the first trimester $(p=0.51)$ and was not estimable for the secondtrimester or combined-trimester analysis owing to the small number of women in some race/ethnic groups.

Sensitivity analyses assessed the impact of missing data with multiple imputations $(M=50)$. Most $(67 \%)$ of the missing data resulted from a lack of medical chart abstraction. There were no significant differences in any of the key variables including age, race/ethnicity, education, pre-pregnancy BMI, social support, stress or depression scores in the first or second trimester according to whether the medical chart was available $(p>0.10)$.

The second set of analyses assessed the association between GDM and risk of postpartum depression. Analyses were adjusted for pre-pregnancy BMI, education and gestational weight gain in the first and second trimesters prior to the diagnosis of GDM. Age and race/ethnicity were accounted for by matching the non-GDM controls to the women with GDM. There was no variation in marital status among women with postpartum depression, and therefore the model with marital status was not estimable. A second model adjusted for firsttrimester depression. To further remove the potential for confounding due to depression before the diagnosis of GDM, sensitivity analyses were conducted by limiting the analysis to women who did not have 'suspected depression' during pregnancy as defined by depression scores $\geq 10$ in the first or second trimesters. All analyses were completed using SAS 
version 9.4 (Cary, NC, USA) with $p$ values $<0.05$ considered significant for main effects and $<0.15$ significant for interactions.

\section{Results}

Association between depressive symptoms in the first and second trimester and GDM Average first-trimester depressive symptom scores were 5.1 (SD 3.9), with $13.4 \%$ of women meeting the threshold for suspected depression. Depression scores varied across many characteristics, including race/ethnicity, education, marital status, social support and stress (Table 1). Depressive symptoms were slightly lower in the second trimester, by 0.6 points. The average secondtrimester score was 4.4 (SD 3.8), with suspected depression in $9.8 \%$ of women. Second-trimester scores similarly varied across maternal characteristics, with one exception - that women in the highest quartile were slightly younger. Among parous women, depression scores in either trimester did not differ in women with a history of GDM $(n=25)$.

Women with first-trimester depression scores in the highest quartile experienced a twofold (95\% CI 1.06, 3.78) significantly increased risk of GDM compared with women in the lowest quartile (Table 2). The association was slightly attenuated to 1.72 -fold $(95 \%$ CI $0.92,3.23)$ after adjusting for relevant covariates. A similar pattern was observed with second-trimester depression scores.

The association between depression and GDM was stronger among non-obese women and null among women with obesity ( $p$ for interaction: first trimester $=0.11$; second trimester $=0.03)($ Table 3$)$. Even after adjustment, there was a significant 2.81 -fold $(95 \%$ CI $1.17,6.73)$ increased risk of GDM among non-obese women in the highest quartile of firsttrimester symptoms, with a significant linear trend of increasing GDM risk with increasing depressive symptoms ( $p$ for linear trend $=0.02$ ). A significant and positive association between increasing second-trimester symptoms and risk of GDM was also observed ( $p$ for linear trend $=0.01$ ), although the magnitude of association was less strong than for the first trimester.

Compared with women who remained in the lowest quartile in the first and second trimesters, women with elevated depressive symptoms in both trimesters (always quartile 4) had a significant 3.21 -fold $(95 \%$ CI $1.00,10.28)$ increased risk of GDM (Table 4). This risk was even stronger, although

Table 1 Maternal characteristics by quartiles of depressive symptom score in the first and second trimesters for the NICHD Fetal Growth StudiesSingleton Cohort (2009-2013)

\begin{tabular}{|c|c|c|c|c|c|c|c|c|c|c|c|}
\hline \multirow[t]{2}{*}{ Variable } & \multirow[b]{2}{*}{$n$} & \multicolumn{4}{|c|}{ Trimester 1 depression score ( $8-13$ weeks) } & \multirow[b]{2}{*}{$p$ values } & \multicolumn{4}{|c|}{$\begin{array}{l}\text { Trimester } 2 \text { depression score } \\
(16-22 \text { weeks })^{\mathrm{a}}\end{array}$} & \multirow[b]{2}{*}{$p$ values } \\
\hline & & $\begin{array}{l}\text { Quartile } 1 \\
(n=474) \\
\%\end{array}$ & $\begin{array}{l}\text { Quartile } 2 \\
(n=523) \\
\%\end{array}$ & $\begin{array}{l}\text { Quartile } 3 \\
(n=691) \\
\%\end{array}$ & $\begin{array}{l}\text { Quartile } 4 \\
(n=789) \\
\%\end{array}$ & & $\begin{array}{l}\text { Quartile } 1 \\
(n=388) \\
\%\end{array}$ & $\begin{array}{l}\text { Quartile } 2 \\
(n=791) \\
\%\end{array}$ & $\begin{array}{l}\text { Quartile } 3 \\
(n=655) \\
\%\end{array}$ & $\begin{array}{l}\text { Quartile } 4 \\
(n=638) \\
\%\end{array}$ & \\
\hline Age (years) ${ }^{\mathrm{b}}$ & 2477 & $28.4(5.5)$ & $28.4(5.4)$ & $28.0(5.4)$ & $27.9(5.6)$ & 0.28 & $28.0(5.5)$ & $28.7(5.5)$ & $27.9(5.5)$ & $27.8(5.5)$ & 0.01 \\
\hline Race/ethnicity & & & & & & $<0.001$ & & & & & $<0.001$ \\
\hline Non-Hispanic white & 694 & 35.4 & 33.3 & 28.8 & 19.4 & & 35.8 & 30.7 & 28.1 & 19.9 & \\
\hline Non-Hispanic black & 683 & 27.4 & 27.5 & 25.8 & 29.3 & & 24.5 & 25.4 & 26.9 & 33.1 & \\
\hline Hispanic & 713 & 28.7 & 27.5 & 28.4 & 30.0 & & 29.6 & 30.2 & 29.0 & 26.3 & \\
\hline Asian & 387 & 8.4 & 11.7 & 17.1 & 21.3 & & 10.1 & 13.7 & 16.0 & 20.7 & \\
\hline Pre-pregnancy BMI $\left(\mathrm{kg} / \mathrm{m}^{2}\right)^{\mathrm{b}}$ & 2477 & $25.1(2.1)$ & $25.6(2.5)$ & $25.6(2.9)$ & $25.5(3.0)$ & 0.42 & $25.2(1.9)$ & $25.5(3.0)$ & $25.4(2.7)$ & $25.7(2.8)$ & 0.48 \\
\hline Normal weight & 1397 & 58.2 & 57.0 & 55.1 & 56.0 & 0.59 & 58.8 & 56.8 & 56.9 & 54.2 & 0.45 \\
\hline Overweight & 651 & 27.2 & 25.4 & 27.5 & 25.2 & & 26.8 & 26.7 & 24.3 & 27.3 & \\
\hline Obese & 429 & 14.6 & 17.6 & 17.4 & 18.8 & & 14.4 & 16.6 & 18.8 & 18.5 & \\
\hline Education & & & & & & $<0.001$ & & & & & $<0.001$ \\
\hline High school or less & 720 & 26.8 & 24.9 & 26.6 & 35.4 & & 28.4 & 25.7 & 30.2 & 32.6 & \\
\hline $\begin{array}{l}\text { Some college or associate } \\
\text { degree }\end{array}$ & 752 & 27.4 & 31.2 & 31.8 & 30.3 & & 29.1 & 28.7 & 29.0 & 34.5 & \\
\hline Bachelor's degree or higher & 1005 & 45.8 & 44.0 & 41.5 & 34.3 & & 42.5 & 45.6 & 40.8 & 32.9 & \\
\hline Nulliparous & 1168 & 44.5 & 43.8 & 51.8 & 46.9 & 0.02 & 46.4 & 47.9 & 47.9 & 45.9 & 0.84 \\
\hline Married or living with partner & 1843 & 79.7 & 80.5 & 74.1 & 67.4 & $<0.001$ & 78.6 & 78.5 & 72.4 & 68.7 & $<0.001$ \\
\hline Full-time job or student & 1759 & 73.2 & 71.1 & 72.5 & 68.3 & 0.20 & 74.0 & 72.3 & 70.8 & 68.0 & 0.17 \\
\hline $\mathrm{GDM}^{\text {in a prior pregnancy }}{ }^{\mathrm{c}}$ & 25 & 0.8 & 1.0 & 0.7 & 1.4 & 0.63 & 0.8 & 0.8 & 1.4 & 1.1 & 0.64 \\
\hline Social support, $8-13$ weeks $^{\mathrm{b}}$ & 2477 & $23.8(2.0)$ & $23.4(2.3)$ & $22.7(2.7)$ & $20.8(4.5)$ & $<0.001$ & $23.6(2.3)$ & $23.1(2.6)$ & $22.3(3.3)$ & $21.0(4.4)$ & $<0.001$ \\
\hline Stress, $8-13$ weeks $^{\mathrm{b}}$ & 2477 & $6.2(4.5)$ & $8.7(4.5)$ & $11.7(4.6)$ & $16.3(5.7)$ & $<0.001$ & $7.0(5.3)$ & $9.3(5.3)$ & $12.5(5.1)$ & $15.8(5.7)$ & $<0.001$ \\
\hline GDM & 105 & 2.5 & 4.2 & 4.5 & 5.1 & 0.18 & 3.1 & 3.8 & 3.7 & 5.3 & 0.26 \\
\hline
\end{tabular}

${ }^{a}$ Women diagnosed with GDM prior to the second-trimester depression assessment were excluded $(n=5)$

${ }^{\mathrm{b}}$ Values are presented as mean $\pm \mathrm{SD}$

${ }^{\mathrm{c}}$ Values reported are for non-nulliparous women 
Table 2 Association between depressive symptoms in the first and second trimesters and subsequent risk of GDM in the NICHD Fetal Growth Studies-Singleton Cohort (2009-2013)

\begin{tabular}{|c|c|c|c|c|c|}
\hline Variable & $\begin{array}{l}\text { Depression scores } \\
\text { median (range) }\end{array}$ & $n$ total & $\%$ GDM & $\begin{array}{l}\text { Unadjusted RR } \\
(95 \% \mathrm{CI})\end{array}$ & Adjusted RR $(95 \% \mathrm{CI})^{\mathrm{a}}$ \\
\hline First trimester $^{\mathrm{b}}$ & & & & $p$ for trend $=0.04$ & $p$ for trend $=0.11$ \\
\hline Quartile 1 & $0(0-1)$ & 474 & 2.5 & 1.00 (reference) & 1.00 (reference) \\
\hline Quartile 2 & $2(2-3)$ & 523 & 4.2 & $1.66(0.83,3.32)$ & $1.44(0.73,2.84)$ \\
\hline Quartile 3 & $5(4-6)$ & 691 & 4.5 & $1.77(0.92,3.42)$ & $1.53(0.80,2.94)$ \\
\hline Quartile 4 & $9(7-27)$ & 789 & 5.1 & $2.00(1.06,3.78)$ & $1.72(0.92,3.23)$ \\
\hline Second trimester ${ }^{\mathrm{b}, \mathrm{c}}$ & & & & $p$ for trend $=0.08$ & $p$ for trend $=0.13$ \\
\hline Quartile 1 & $0(0-0)$ & 388 & 3.1 & 1.00 (reference) & 1.00 (reference) \\
\hline Quartile 2 & $2(1-3)$ & 791 & 3.8 & $1.23(0.63,2.37)$ & $1.21(0.64,2.29)$ \\
\hline Quartile 3 & $5(4-6)$ & 655 & 3.7 & $1.18(0.60,2.34)$ & $1.19(0.61,2.33)$ \\
\hline Quartile 4 & $9(7-23)$ & 638 & 5.3 & $1.72(0.90,3.29)$ & $1.60(0.85,3.00)$ \\
\hline
\end{tabular}

${ }^{\text {a }}$ Model adjusted for maternal age, race (white, black, Hispanic, Asian), education (high school or less, some college or associate degree, bachelor's degree or higher), marital status and pre-pregnancy BMI

${ }^{\mathrm{b}}$ First trimester assessed at study enrolment at 8-13 weeks. Second trimester assessed at 16-22 weeks

${ }^{\mathrm{c}}$ Five women (four non-obese, one obese) were diagnosed early in the second trimester before the study visit and evaluation of depression, and were therefore excluded from the second-trimester models not significant, when limited only to non-obese women (RR 4.02, 95\% CI 0.95, 16.97). All results were similar after excluding the women who reported taking antidepressant medications at the second-trimester visit $(n=2)$ (data not shown).

\section{Association between GDM and postpartum depression} Women with GDM had a significant fourfold $(95 \%$ CI 1.17 , 13.65) greater risk of postpartum depression. This risk remained high and significant after adjustment for covariates including first-trimester depressive symptoms (RR 4.62, 95\% CI 1.26, 16.98) (Table 5). The findings were similar after excluding women with suspected depression in the first trimester $(n=142)$ (RR 4.26, 95\% CI 1.15, 15.76). Furthermore, the magnitude of the adjusted association was similar when the outcome of suspected depression was based only on depressive symptoms $(n=13)$ and postpartum medication use was not considered (RR 4.23, 95\% CI 1.12, 15.98). No women in the postpartum sample were taking antidepressant medication during pregnancy.

Table 3 Association between depressive symptoms in the first and second trimesters before the diagnosis of GDM and GDM risk by pre-pregnancy obesity status in the NICHD Fetal Growth Studies-Singleton Cohort (2009-2013)

\begin{tabular}{|c|c|c|c|c|c|c|c|c|}
\hline \multirow[t]{2}{*}{ Variable } & \multicolumn{4}{|l|}{ Non-obese } & \multicolumn{4}{|l|}{ Obese } \\
\hline & $\begin{array}{l}\text { Depression score } \\
\text { median (range) }\end{array}$ & $n$ total & $\%$ GDM & Adjusted RR $(95 \% \mathrm{CI})^{\mathrm{a}}$ & $\begin{array}{l}\text { Depression score } \\
\text { median (range) }\end{array}$ & $n$ total & $\%$ GDM & $\begin{array}{l}\text { Adjusted RR } \\
(95 \% \mathrm{CI})^{\mathrm{a}}\end{array}$ \\
\hline First trimester $^{\mathrm{b}}$ & & & & $p$ for trend $=0.02$ & & & & $p$ for trend $=0.40$ \\
\hline Quartile 1 & $0(0-1)$ & 405 & 1.5 & 1.00 (reference) & $0(0-1)$ & 69 & 8.7 & 1.00 (reference) \\
\hline Quartile 2 & $2(2-3)$ & 431 & 3.0 & $1.97(0.77,5.04)$ & $3(2-4)$ & 137 & 10.9 & $1.09(0.44,2.68)$ \\
\hline Quartile 3 & $5(4-6)$ & 571 & 3.5 & $2.12(0.86,5.24)$ & $6(5-7)$ & 107 & 6.5 & $0.82(0.29,2.31)$ \\
\hline Quartile 4 & $9(7-21)$ & 641 & 4.8 & $2.81(1.17,6.73)$ & $9.5(8-27)$ & 116 & 6.0 & $0.74(0.26,2.12)$ \\
\hline Second trimester ${ }^{\mathrm{b}, \mathrm{c}}$ & & & & $p$ for trend $=0.01$ & & & & $p$ for trend $=0.27$ \\
\hline Quartile 1 & $0(0-0)$ & 332 & 1.8 & 1.00 (reference) & $0(0-1)$ & 92 & 12.0 & 1.00 (reference) \\
\hline Quartile 2 & $2(1-3)$ & 660 & 2.4 & $1.27(0.51,3.17)$ & $3(2-3)$ & 95 & 9.5 & $0.88(0.38,2.03)$ \\
\hline Quartile 3 & $5(4-6)$ & 532 & 3.2 & $1.73(0.69,4.35)$ & $5(4-6)$ & 123 & 5.7 & $0.59(0.24,1.43)$ \\
\hline Quartile 4 & $9(7-23)$ & 520 & 5.2 & $2.38(0.99,5.69)$ & $9(7-20)$ & 118 & 5.9 & $0.64(0.26,1.57)$ \\
\hline
\end{tabular}

${ }^{a}$ Model adjusted for maternal age, race (white, black, Hispanic, Asian), education (high school or less, some college or associate degree, bachelor's degree or higher), marital status and pre-pregnancy BMI

${ }^{\mathrm{b}}$ First trimester assessed at study enrolment at 8-13 weeks. Second trimester assessed at 16-22 weeks

${ }^{\mathrm{c}}$ Five women (four non-obese, one obese) were diagnosed early in the second trimester before the study visit and evaluation of depression, and were therefore excluded from the second-trimester models 
Table 4 Association between depression symptoms in the first and second trimester and subsequent risk of gestational diabetes in the NICHD Fetal Growth Studies-Singleton Cohort (2009-2013)

\begin{tabular}{|c|c|c|c|c|c|c|c|c|c|}
\hline \multirow{2}{*}{$\begin{array}{l}\text { Depression scores in the } \\
\text { first and second trimesters }\end{array}$} & \multicolumn{3}{|l|}{ Overall } & \multicolumn{3}{|c|}{ Non-obese } & \multicolumn{3}{|l|}{ Obese } \\
\hline & $n$ total & $\%$ GDM & $\begin{array}{l}\text { Adjusted RR } \\
(95 \% \mathrm{CI})^{\mathrm{b}}\end{array}$ & $n$ total & $\%$ GDM & $\begin{array}{l}\text { Adjusted RR } \\
(95 \% \mathrm{CI})^{\mathrm{b}}\end{array}$ & $n$ total & $\%$ GDM & $\begin{array}{l}\text { Adjusted RR } \\
(95 \% \mathrm{CI})^{\mathrm{b}}\end{array}$ \\
\hline Both trimesters Q1 & 212 & 1.4 & 1.00 (reference) & 186 & 1.1 & 1.00 (reference) & 36 & 11.1 & 1.00 (reference) \\
\hline Ever in Q2 & 565 & 3.9 & $2.28(0.71,7.35)$ & 476 & 2.3 & $2.00(0.46,8.79)$ & 100 & 12.0 & $0.83(0.29,2.36)$ \\
\hline Ever in Q3 & 692 & 3.8 & $2.21(0.69,7.07)$ & 560 & 2.5 & $2.16(0.50,9.29)$ & 127 & 7.1 & $0.66(0.22,2.00)$ \\
\hline Ever in Q4 & 580 & 4.3 & $2.45(0.76,7.88)$ & 484 & 4.3 & $3.40(0.81,14.36)$ & 96 & 4.2 & $0.36(0.11,1.24)$ \\
\hline Both trimesters Q4 & 423 & 5.7 & $3.21(1.00,10.28)$ & 338 & 5.3 & $4.02(0.95,16.97)$ & 69 & 7.2 & $0.72(0.21,2.49)$ \\
\hline
\end{tabular}

${ }^{\text {a }}$ Five women (four non-obese, one obese) were diagnosed early in the second trimester before the study visit and evaluation of depression, and were therefore excluded from the models

${ }^{\mathrm{b}}$ Model adjusted for maternal age, race (white, black, Hispanic, Asian), education (high school or less, some college or associate degree, bachelor's degree or higher), marital status and pre-pregnancy BMI

\section{Discussion}

In this prospective cohort study, we observed a bidirectional association between depression and GDM throughout pregnancy and the postpartum period. Using longitudinal assessments, we delineated the temporality of these relations such that depression early in pregnancy was significantly associated with an increased risk of GDM, and GDM was strongly associated with an increased risk of postpartum depression. Moreover, the risk of GDM was strongest among non-obese women and when depressive symptoms were increased in the first trimester and persisted into the second trimester.

Pregnant women are at high risk of developing depressive symptoms, with at least one in ten US women suffering from depression during pregnancy [26]. Depression in pregnancy may increase the risk of adverse pregnancy outcomes. Specifically, our findings confirm and extend previous limited studies showing that depression is related to an increased risk of GDM $[9,10]$. For instance, in a study based on medical record databases, a medical history of depression was associated with an approximately $40 \%$ increased risk of GDM [9]. However, in that study it was unclear whether women had active depressive symptoms during pregnancy, and symptom severity could not be assessed. In the present study, we specifically examined women without a medical history of mental health conditions who had active depressive symptoms during pregnancy. Another previous study found that any depression during, but not prior to, pregnancy was associated with GDM, but this finding did not persist after accounting for other major risk factors [10]. This retrospective study could not distinguish between depression during pregnancy that occurred before the diagnosis of GDM and depression that may have been a consequence of GDM. Through longitudinal ascertainment, we have shown that persistent depressive symptoms prior to a diagnosis of GDM increase the risk of GDM. Another new finding identified in our study was that the association between depression and GDM was strongest among nonobese women.

Our study highlights the pregnancy and postpartum periods, when women have an increased susceptibility to developing depressive symptoms, as an important window during the life course for the interplay between depression and glucose intolerance. Such findings are biologically plausible. Melancholic depression can activate the sympathetic nervous system and the hypothalamic-pituitary-adrenal axis, leading to increased glucocorticoid and cortisol levels [27]. This in turn leads to downstream effects on the liver and adipose tissue, increasing circulating levels of non-esterified fatty acids and glucose and subsequently leading to insulin resistance and GDM [28].

Table 5 Association between GDM and postpartum depression assessed at approximately 6 weeks postpartum for the NICHD Fetal Growth StudiesSingleton Cohort (2009-2013)

\begin{tabular}{lllll}
\hline GDM & $\begin{array}{l}\text { Postpartum depression/ } \\
\text { total }(n)\end{array}$ & $\begin{array}{l}\text { Unadjusted } \\
\text { RR }(95 \% \mathrm{CI})\end{array}$ & $\begin{array}{l}\text { Adjusted model A } \\
\text { RR }(95 \% \mathrm{CI})\end{array}$ & $\begin{array}{l}\text { Adjusted model B } \\
\text { RR }(95 \% \mathrm{CI})\end{array}$ \\
\hline No & $3 / 81$ & 1.00 (reference) & 1.00 (reference) & 1.00 (reference) \\
Yes & $12 / 81$ & $4.00(1.17,13.65)$ & $4.87(1.36,17.48)$ & $4.62(1.26,16.98)$ \\
\hline
\end{tabular}

${ }^{\text {a }}$ Model A was adjusted for pre-pregnancy BMI, education (high school or less, some college or associate degree, bachelor's degree or higher), and firstand second-trimester gestational weight gain. Controls without GDM were matched 1:1 to GDM cases according to age and race/ethnicity

${ }^{\mathrm{b}}$ Model B was adjusted for all the covariates in model A as well as first-trimester depression scores 
Interestingly, the association between depression and GDM in our study was observed primarily among non-obese women, even though, consistent with prior literature [29], obese women tended to have higher depression scores than non-obese women. Although it is possible that the baseline elevated risk of GDM among women with obesity may mask the variation observed with increasing depressive symptoms, an alternative hypothesis is that the subtype of depression early in pregnancy may be different between non-obese and obese women. Individuals with obesity are more likely to have depression with atypical features [30]. Individuals with atypical depression show low activity of the hypothalamicpituitary-adrenal axis, lower cortisol levels and higher levels of inflammation [31,32]. Therefore atypical depression may affect the risk of GDM through less direct behavioural mechanisms (e.g. increased appetite), and it is possible that this may be less apparent among women with obesity. Unfortunately, in this study we cannot distinguish between heterogeneous subtypes of depression as we assessed only depressive symptoms and do not have diagnoses of clinical depression. Further examination of metabolic changes early in pregnancy among women with depression may help to understand this difference in risk profile between obese and non-obese women.

Our study is unique in that a subset of the sample was followed postpartum, allowing for assessment of the association between GDM and depression at approximately 6 weeks postpartum. We found that GDM substantially increased a woman's risk of postpartum depression approximately fourfold. Our findings in a low-risk, diverse, US sample confirm those of a prior study from Iran [15]. Interestingly, other studies of the association between GDM and postpartum depression have been null, but these studies either assessed depression much later, at 6 months postpartum [12], or used a selfreport of depression [13], unlike our study, which used a validated measure of depressive symptoms at 6 weeks postpartum.

In addition to the major life changes that occur with childbirth, women undergo substantial hormonal changes from pregnancy to the postpartum period, increasing their potential for metabolic dysregulation and depression. The altered metabolic state associated with GDM could further impact neurological functions or hormonal changes that occur postpartum, potentially increasing the risk of depression [33, 34]. For example, maternal adipokine concentrations are increased in women with GDM [28], even in the third trimester, and increased inflammation is associated with an increased risk of atypical depression, possibly the subtype of depression more commonly seen in the postpartum period $[31,35]$. Alternatively, the mechanism may relate to the psychological stress associated with factors downstream of GDM, such as caesarean delivery or added stress from neonatal complications [36]. Of note, previous randomised controlled trials of GDM treatment vs routine care have found that postpartum depression is decreased with treatment of GDM [37, 38], which supports a potential aetiological role of hyperglycaemia in the development of depression, and that the association between GDM and postpartum depression can be mitigated with GDM treatment.

The prospective, well-defined nature of GDM and depressive symptoms in this longitudinal cohort was a major strength of our study. GDM was characterised based on a review of medical records, and depression was symptomatically assessed using a validated scale that is used clinically to screen for depression and refer women for further evaluation, extending the clinical relevance of our findings [19]. This study was conducted with a multiracial cohort from across the US, with generalisability to women without chronic conditions prior to pregnancy. Although these inclusion criteria may potentially limit the power of our study, as a relatively small number of women developed GDM, it highlights that depressive symptoms are prevalent even among women considered to be of low risk. We reviewed self-reported medication data and confirmed that only two women were taking antidepressant medications before the diagnosis of GDM, reducing the risk for confounding due to this treatment. Much of the missing data in our study resulted from the missing medical chart abstraction $(7.8 \%)$. We performed multiple imputation analyses to assess for bias owing to the small amount of missing data in our analysis of the effect of depression on risk of GDM and observed consistent results. We considered many important risk factors, including measures of perceived social support and stress, although these were not included in the analysis as they were not associated with risk of GDM.

Conclusions Through the use of longitudinal prospective data, our findings delineate the temporal relations between GDM and depression during the critical pregnancy and postpartum periods. The American College of Obstetricians and Gynecologists recommends that all women are screened for depression at least once during pregnancy or in the postpartum period [19]. Our data suggest that screening early in pregnancy may be particularly important even among women without a history of mental health conditions. In addition, women with GDM may require closer monitoring postpartum given their elevated risk of postpartum depression. Taken together, our work extends previous data supporting depression and glucose intolerance as comorbid conditions among nonpregnant individuals to women during pregnancy and postpartum, a critical time window for the health and well-being of both women and their offspring.

Funding This work was supported by the Intramural Research Program of the Eunice Kennedy Shriver NICHD, National Institutes of Health and through ARRA funding (contracts: HHSN275200800013C, HHSN275200800002I, HHSN27500006, HHSN275200800003IC, 
HHSN275200800014C, HHSN275200800012C, HHSN275200800028C, HHSN275201000009C)

Duality of interest statement The authors declare that there is no duality of interest associated with this manuscript.

Contribution statement $\mathrm{SNH}$ and $\mathrm{CZ}$ had full access to all of the data in the study and take responsibility for the integrity of the data and the accuracy of the data analysis. The design and conduct of the study was carried out by CZ, PSA and GMBL The study analysis concept and design was carried out by $\mathrm{SNH}$ and CZ. Data preparation and statistical analyses were carried out by $\mathrm{SNH}, \mathrm{CZ}$ and PSA. Interpretation of results was carried out by SNH, CZ, YZ, SR and PSA. SNH and CZ wrote the paper and critical revision of the paper was carried out by $\mathrm{SNH}, \mathrm{CZ}, \mathrm{YZ}$, SR, GMBL and PSA. All authors have approved the final version of the manuscript.

\section{References}

1. Shim RS, Baltrus P, Ye J, Rust G (2011) Prevalence, treatment, and control of depressive symptoms in the United States: results from the National Health and Nutrition Examination Survey (NHANES), 2005-2008. J Am Board Fam Med 24:33-38

2. Moulton CD, Pickup JC, Ismail K (2015) The link between depression and diabetes: the search for shared mechanisms. Lancet Diabetes Endocrinol 3:461-471

3. Chen PC, Chan YT, Chen HF, Ko MC, Li CY (2013) Populationbased cohort analyses of the bidirectional relationship between type 2 diabetes and depression. Diabetes Care 36:376-382

4. Golden SH, Lazo M, Carnethon M et al (2008) Examining a bidirectional association between depressive symptoms and diabetes. JAMA 299:2751-2759

5. Pan A, Lucas M, Sun Q et al (2010) Bidirectional association between depression and type 2 diabetes mellitus in women. Arch Intern Med 170:1884-1891

6. Rustad JK, Musselman DL, Nemeroff CB (2011) The relationship of depression and diabetes Pathophysiological and treatment implications. Psychoneuroendocrinology 36:1276-1286

7. Lustman PJ, Clouse RE (2005) Depression in diabetic patients. The relationship between mood and glycemic control. J Diabetes Complicat 19:113-122

8. Snoek FJ, Bremmer MA, Hermanns N (2015) Constructs of depression and distress in diabetes: time for an appraisal. Lancet Diabetes Endocrinol 3:450-460

9. Bowers K, Laughon SK, Kim S et al (2013) The association between a medical history of depression and gestational diabetes in a large multi-ethnic cohort in the United States. Paediatr Perinat Epidemiol 27:323-328

10. Wilson BLDJ, Latendresse G, Wong B, Baksh L (2015) Exploring the psychosocial predictors of gestational diabetes and birth weight. J Obstet Gynecol Neonatal Nurs 44:760-771

11. Morrison C, McCook JG, Bailey BA (2015) First trimester depression scores predict development of gestational diabetes mellitus in pregnant rural Appalachian women. J Psychosom Obstet Gynaecol $37: 21-25$

12. Huang T, Rifas-Shiman SL, Ertel KA et al (2015) Pregnancy hyperglycaemia and risk of prenatal and postpartum depressive symptoms. Paediatr Perinat Epidemiol 29:281-289

13. Liu CH, Tronick E (2013) Rates and predictors of postpartum depression by race and ethnicity: results from the 2004 to $2007 \mathrm{New}$
York City PRAMS survey (Pregnancy Risk Assessment Monitoring System). Matern Child Health J 17:1599-1610

14. Kim C, Brawarsky P, Jackson RA, Fuentes-Afflick E, Haas JS (2005) Changes in health status experienced by women with gestational diabetes and pregnancy-induced hypertensive disorders. J Womens Health (Larchmt) 14:729-736

15. Abdollahi F, Zarghami M, Azhar MZ, Sazlina SG, Lye MS (2014) Predictors and incidence of post-partum depression: a longitudinal cohort study. J Obstet Gynaecol Res 40:2191-2200

16. Kozhimannil KB, Pereira MA, Harlow BL (2009) Association between diabetes and perinatal depression among low-income mothers. JAMA 301:842-847

17. Buck Louis GM, Grewal J, Albert PS et al (2015) Racial/ethnic standards for fetal growth: the NICHD Fetal Growth Studies. Am J Obstet Gynecol 213:449.e1-449.e41

18. Cox JL, Holden JM, Sagovsky R (1987) Detection of postnatal depression. Development of the 10-item Edinburgh Postnatal Depression Scale. Br J Psychiatry 150:782-786

19. American College of Obstetricians and Gynecologists (2015) Screening for perinatal depression. Committee Opinion No. 630. Obstet Gynecol 125:1268-1271

20. American College of Obstetricians and Gynecologists (2001) Clinical management guidelines for obstetrician-gynecologists. Number 30, September 2001 (replaces technical bulletin number 200, December 1994). Gestational diabetes. Obstet Gynecol 98: $525-538$

21. Elsenbruch S, Benson S, Rücke M et al (2007) Social support during pregnancy: effects on maternal depressive symptoms, smoking and pregnancy outcome. Hum Reprod 22:869-877

22. Roy-Matton N, Moutquin J-M, Brown C, Carrier N, Bell L (2011) The impact of perceived maternal stress and other psychosocial risk factors on pregnancy complications. J Obstet Gynaecol Can 33: 344-352

23. Cohen S, Kamarck T, Mermelstein R (1983) A global measure of perceived stress. J Health Soc Behav 24:385-396

24. Vaglio J Jr, Conard M, Poston WS et al (2004) Testing the performance of the ENRICHD Social Support Instrument in cardiac patients. Health Qual Life Outcomes 2:24

25. Zou G (2004) A modified Poisson regression approach to prospective studies with binary data. Am J Epidemiol 159:702-706

26. Silveira ML, Whitcomb BW, Pekow P, Carbone ET, Chasan-Taber L (2016) Anxiety, depression, and oral health among US pregnant women: 2010 Behavioral Risk Factor Surveillance System. J Public Health Dent 76:56-64

27. Musselman DL, Betan E, Larsen H, Phillips LS (2003) Relationship of depression to diabetes types 1 and 2: epidemiology, biology, and treatment. Biol Psychiatry 54:317-329

28. Fasshauer M, Bluher M, Stumvoll M (2014) Adipokines in gestational diabetes. Lancet Diabetes Endocrinol 2:488-499

29. Luppino FS, de Wit LM, Bouvy PF et al (2010) Overweight, obesity, and depression: a systematic review and meta-analysis of longitudinal studies. Arch Gen Psychiatry 67:220-229

30. Lasserre AM, Glaus J, Vandeleur CL et al (2014) Depression with atypical features and increase in obesity, body mass index, waist circumference, and fat mass: a prospective, population-based study. JAMA Psychiatry 71:880-888

31. Lamers F, Vogelzangs N, Merikangas K, De Jonge P, Beekman A, Penninx B (2013) Evidence for a differential role of HPA-axis function, inflammation and metabolic syndrome in melancholic versus atypical depression. Mol Psychiatry 18:692-699

32. Thase ME (2007) Recognition and diagnosis of atypical depression. J Clin Psychiatry 68:1,478-416

33. O'Hara MW, Wisner KL (2014) Perinatal mental illness: definition, description and aetiology. Pract Res Clin Obstet Gynaecol 28:3-12 
34. Sandu RE, Buga AM, Uzoni A, Petcu EB, Popa-Wagner A (2015) Neuroinflammation and comorbidities are frequently ignored factors in CNS pathology. Neural Regen Res 10:1349-1355

35. Kammerer M, Taylor A, Glover V (2006) The HPA axis and perinatal depression: a hypothesis. Arch Womens Ment Health 9:187196

36. Catalano PM, McIntyre HD, Cruickshank JK et al (2012) The hyperglycemia and adverse pregnancy outcome study: associations of
GDM and obesity with pregnancy outcomes. Diabetes Care 35: 780-786

37. Beucher G, Viaris de Lesegno B, Dreyfus M (2010) Maternal outcome of gestational diabetes mellitus. Diabetes Metab 36:522-537

38. Crowther CA, Hiller JE, Moss JR, McPhee AJ, Jeffries WS, Robinson JS, Australian Carbohydrate Intolerance Study in Pregnant Women (ACHOIS) Trial Group (2005) Effect of treatment of gestational diabetes mellitus on pregnancy outcomes. N Engl J Med 352:2477-2486 